\title{
Adsorption of large hydrocarbons on coinage metals: a van der Waals density functional study
}

Jonas Björk and Sven Stafström

\section{Linköping University Post Print}

\section{Tweet}

N.B.: When citing this work, cite the original article.

Original Publication:

Jonas Björk and Sven Stafström, Adsorption of large hydrocarbons on coinage metals: a van der Waals density functional study, 2014, ChemPhysChem, (15), 13, 2851-2858.

http://dx.doi.org/10.1002/cphc.201402063

Copyright: Wiley-VCH Verlag http://www.wiley-vch.de/publish/en/

Postprint available at: Linköping University Electronic Press

http://urn.kb.se/resolve?urn=urn:nbn:se:liu:diva-107770 


\title{
Adsorption of large hydrocarbons on coinage metals: a van der Waals density functional study
}

\author{
Jonas Björk and Sven Stafström \\ Department of Physics, Chemistry and Biology, IFM, Linköping University, Linköping, 581 \\ 83, Sweden
}

May 22, 2014

\begin{abstract}
We study the adsorption of organic molecules on the close-packed facets of the coinage metals, illustrating how accurately adsorption heights can be described using recent advances of the van der Waals density functional (vdWDF), with the so called optPBE/vdWDF, optB86b/vdWDF, vdWDF2 and rev/vdWDF2 functionals. The adsorption of two prototypical aromatic hydrocarbons is investigated, and the calculated adsorption heights are compared to experimental literature values from normal incident X-ray standing wave absorption as well as a state-of-the art semi-empirical method. It is shown that both the optB86b/vdWDF and rev/vdWDF2 functionals describe adsorption heights with an accuracy of $0.1 \AA$ compared to experimental values, and are concluded as a reliable methods of choice for related systems.
\end{abstract}

\section{Introduction}

The assembly of organic molecules on metal surfaces into one- and two-dimensional nanoarchitectures, stabilized by either non-covalent ${ }^{1-5}$ or covalent interactions, ${ }^{6-10}$ has become a very active area in surface and nanoscale science over the past decade. Density functional theory (DFT) is important as a complemental tool for the experimental characterization of molecular architectures on surfaces. ${ }^{11}$ This is, however, not the only role DFT-based theoretical modeling has to play. During the 
last years it has been frequently used for understanding the underlying surface reactions that are responsible for the formation of many structures. ${ }^{12-16}$ One may even envision the use of DFT in the computational screening of new materials with superior properties for certain applications, ${ }^{16}$ which has already proven powerful in heterogeneous catalysis. ${ }^{17}$

In all of these aspects, it is of the uttermost importance to have an accurate theory that reliably describes the interactions between an organic molecule and a metal surface. As a matter of fact, such a description remains one of the main challenges for DFT. This is due to the fact that, by definition, long-ranged van der Waals (vdW) interactions are not accounted for by conventional semi-local DFT on the generalized gradient approximation (GGA) level of theory. The approximations inherent to GGA are particularly unviable to describe the adsorption of large organic molecules, where vdW forces contribute with a major portion of the overall attractive interaction. ${ }^{18}$

Two schools of thought for treating non-local van der Waals interactions in DFT calculations have emerged. The first introduces an additive atom pairwise potential of the form $C_{6} R^{-6}$, where $C_{6}$ is an atom-specific parameter determining the interaction strength between two atoms, and $R$ is the separation between the two atoms. ${ }^{19,20}$ While this formulation gives the anticipated asymptotic behavior for large separations, the energy diverges for short distances, why a damping function is needed. The so called dispersion corrected DFT (DFT-D) methods have met criticism, as the results depend more on the ad hoc damping function than the physically justified $C_{6}$-parameters. ${ }^{21}$ Furthermore, this approach is particularly challenging for metal surfaces due to difficulties describing the polarizability of a metal with a pairwise additive potential. ${ }^{22}$ The latter problem was recently addressed with the DFT $+\mathrm{vdW}^{\text {surf }}$ method, ${ }^{23}$ which includes the screening of the substrate electrons when determining the $C_{6}$ coefficients.

The second approach is based on introducing a truly non-local density functional. The so called van der Waals density functional (vdWDF) ${ }^{24}$ was constructed to work seamlessly for both large and small separations, thereby avoiding the problem of the damping function. It is, however, a well established fact that the original version of the vdWDF, based on revPBE exchange, ${ }^{25}$ gives too large adsorption distances. ${ }^{26}$ The last years, several improvements have been made to the vdWDF. These include both updating the non-local kernel of the functional, ${ }^{27}$ as well as adopting different types of semi-local exchange. ${ }^{28-30}$ Recently, it was illustrated ${ }^{31}$ that the vdWDF based on an optimized 
form of the Becke86 functional (optB86b/vdWDF) ${ }^{28,29}$ gives excellent results for single molecule adsorption, as well as for two dimensional metal-organic frameworks, on $\mathrm{Cu}(111)$. However, how this functional performs for other molecules and surfaces is still unclear due to the limited number of studies. It has been suggested that optB86b/vdWDF accurately describes the adsorption of a phtalocyanine on $\mathrm{Au}(111),{ }^{32}$ but the conclusions were based on presumption of the experimental adsorption height, without explicit experimental justification.

In this work, we investigate how different vdWDFs describe the adsorption of the 3,4,9,10perylene-tetracarboxylic acid (PTCDA) and diindenoperylene (DIP) molecules on $\mathrm{Au}(111), \mathrm{Ag}(111)$, and $\mathrm{Cu}(111)$. The investigated functionals include optB86b/vdWDF, optPBE/vdWDF, vdWDF2, and rev/vdWDF2. The two former functionals are based on the original vdWDF adapted with different semi-local exchange, while the latter two are based on the updated correlation suggested in Ref. ${ }^{27}$, the so called vdWDF2, with different semi-local exchange. The two molecules, which are illustrated in Fig. 1, were chosen since there exist experimental values of the adsorption heights, measured by normal incident X-ray standing wave (NI-XSW) absorption. ${ }^{33-36}$ The results from the various vdWDFs will also be compared to those obtained with the DFT+vdW ${ }^{\text {surf }}$ method. ${ }^{23,36}$

\section{(a) PTCDA}

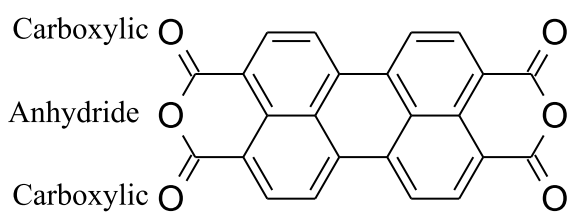

(b) DIP

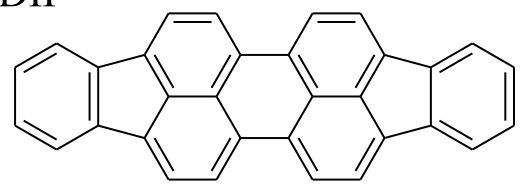

Figure 1 The two molecules considered in this study:

(a) 3,4,9,10-perylene-tetracarboxylic acid $\left(\mathrm{C}_{24} \mathrm{O}_{6} \mathrm{H}_{8}\right.$, PTCDA), and (b) diindenoperylene $\left(\mathrm{C}_{32} \mathrm{H}_{16}\right.$, DIP). For PTCDA the carboxyl and anhydride oxygen atoms are labelled. 


\section{Computational details}

The calculations were performed within the framework of periodic density functional theory (DFT) using the VASP $\operatorname{code}^{37}$ interfaced to the Atomic Simulation Environment (ASE). ${ }^{38}$ Ion-core electron interactions were described using the projected augmented wave method. ${ }^{39,40}$ In all calculations, a $500 \mathrm{eV}$ kinetic energy cutoff was used.

The vdWDF was used to describe all non-local correlation, while local correlation was described by LDA. The exchange was described on the GGA level of theory, using an optimized forms of the Becke 86 (optB86b), and PBE (optPBE). ${ }^{28,29}$ These types of exchange were combined with the nonlocal correlation proposed in the original version of the $\mathrm{vdWDF}^{24}$, resulting in the XC-functionals denoted as optB86b/vdWDF and PBE/vdWDF, respectively. We also performed calculations with the updated version of the $\mathrm{vdWDF}^{27}$, both with the exchange suggested by Lee et al. $^{27}$, denoted as vdWDF2, as well as with a revised form of Becke 86 exchange, recently suggested by Hamada, ${ }^{41}$ denoted as rev/vdWDF2.

The $\mathrm{Cu}(111), \operatorname{Ag}(111)$, and $\mathrm{Au}(111)$ surfaces were modeled by four layered slab separated by at least $15 \AA$ of vacuum. For each of the metal, the calculated lattice constants for respective method were used, which are summarized in the Supporting Information. Structural optimizations were performed until the forces acting on the atoms in adsorbed molecules and the two outermost slab layers were smaller than $0.01 \mathrm{eV} / \AA \AA$.

Adsorption heights were calculated as

$$
h_{\mathrm{ads}}=z_{\mathrm{ads}}-z\left(n^{\text {th }} \text { layer }\right)-(n-1) d_{[111]},
$$

where $z_{\text {ads }}$ is the $z$-coordinate of the molecule, $z$ (n $n^{\text {th }}$ layer $)$ is the $z$-coordinate of the $n^{\text {th }}$ surface layer, and $d_{[111]}$ is the interlayer spacing in the [111] direction. The $n^{\text {th }}$ layer layer is defined as the first fixed layer in the slab; in our case $n=3$. The definition of the adsorption height is illustrated in Fig. 2. We choose this definition as it better relates to NI-XSW measurements, where it is the periodicity between the bulk layers that gives rise to the standing waves. 


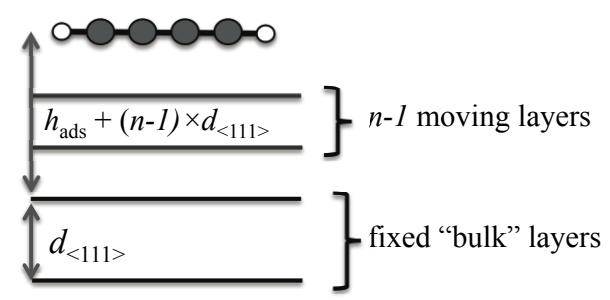

Figure 2 Definition of adsorption height $h_{\mathrm{ads}}$, as obtained from NI-XSW measurements: $h_{\mathrm{ads}}$ is given with respect to the bulk of the substrate.

\subsection{Isolated PTCDA and DIP}

When modeling the adsorption of isolated PTCDA and DIP molecules, for $\mathrm{Cu}(111)$, a surface unit cell of

$$
\left(\begin{array}{cc}
11 & 0 \\
-4 & 8
\end{array}\right)\left(\begin{array}{l}
\mathbf{a}_{1} \\
\mathbf{a}_{2}
\end{array}\right)
$$

was used, while for $\mathrm{Ag}(111)$ and $\mathrm{Au}(111)$, a surface unit cell of

$$
\left(\begin{array}{cc}
10 & 0 \\
-4 & 8
\end{array}\right)\left(\begin{array}{l}
\mathbf{a}_{1} \\
\mathbf{a}_{2}
\end{array}\right)
$$

was used. Here, $\mathbf{a}_{1}$ and $\mathbf{a}_{2}$ are the primitive surface unit cell vectors of respective surface. In the calculations of isolated molecules, a $2 \times 3 k$-point sampling was used, which provides converged adsorption heights and energies within $0.01 \AA$ and $10 \mathrm{meV}$, respectively. In the Supporting Information a part of the convergence check with respect to the $k$-point sampling has been included.

\subsection{Herringbone structure of PTCDA}

For the herringbone structure of PTCDA on $\mathrm{Ag}(111)$ the experimentally determined unit cell, ${ }^{42}$ given by

$$
\left(\begin{array}{cc}
6 & 1 \\
-3 & 5
\end{array}\right)\left(\begin{array}{l}
\mathbf{a}_{1} \\
\mathbf{a}_{2}
\end{array}\right)
$$

was used. On $\mathrm{Au}(111)$ it is well-known that PTCDA does not form a commensurate overlayer, but instead demonstrates a point-on-line growth on the $(22 \times \sqrt{3})$ reconstructed surface. ${ }^{43-45}$ For simplicity, 
for the modeling of the herringbone structure on $\mathrm{Au}(111)$ we use the same unit cell as for $\operatorname{Ag}(111)$, which was suggested in Refs. ${ }^{23,46}$, with the motivation that the lattice vectors of Au and Ag are almost identical.

For the herringbone structure on $\mathrm{Cu}(111)$, two different unit cells can be found in the literature. From scanning tunneling microscopy experiments ${ }^{47}$ a unit cell of

$$
\left(\begin{array}{cc}
5 & 5 \\
-5 & 5
\end{array}\right)\left(\begin{array}{l}
\mathbf{a}_{1} \\
\mathbf{a}_{2}
\end{array}\right)
$$

was proposed. However, Romaner et al. suggested ${ }^{46}$ the alternative unit cel

$$
\left(\begin{array}{cc}
6 & 2 \\
-4 & 6
\end{array}\right)\left(\begin{array}{l}
\mathbf{a}_{1} \\
\mathbf{a}_{2}
\end{array}\right) .
$$

The results presented here were obtained with the latter unit cell, as this was found to give a more stable network than the former (see the Supporting Information for further information). For the herringbone networks a $3 \times 5 k$-point sampling was used, which corresponds to the same accuracy of the $k$-point sampling used for the isolated molecules.

\section{Results}

\subsection{Adsorption of PTCDA}

Fig. 3a-c illustrates the most stable adsorption configurations for an isolated PTCDA molecule on $\mathrm{Cu}(111), \operatorname{Ag}(111)$, and $\mathrm{Au}(111)$, respectively. On $\operatorname{Ag}(111)$ and $\mathrm{Au}(111)$ the molecule is centered above a bridge site, with the long axis of the molecule parallel to the [110] direction; the Bridge- $0^{\circ}$ site. On $\mathrm{Cu}(111)$ on the other hand, optB86b/vdWDF suggests an adsorption geometry where the molecule is centered above a bridge site with its long-axis rotated $30^{\circ}$ away from the [1 10$]$ direction (Bridge- $30^{\circ}$ ), while all other considered methods find an adsorption site where PTCDA is centered above a top site and with its long axis parallel to the [110] direction (Top- $0^{\circ}$ site). For clarity, both the adsorption configurations on $\mathrm{Cu}(111)$ are indicated in Fig. 3. Notably, an important aspect of the adsorption of an isolated DPDI molecule on all the three surfaces is that the carboxyl oxygens sits on-top of a surface atom. For the Bridge- $30^{\circ}$ configuration on $\mathrm{Cu}(111)$, shown in Fig. 3a, also the 
anhydride oxygen is positioned above a top site, at the cost that the entire molecular backbone is closer to the surface. The interface between PTCDA and $\mathrm{Cu}(111)$ presents a delicate mixture of interactions, which will become even more prominent when discussing the herringbone network of PTCDA on this surface. The adsorption data for the most stable adsorption configurations obtained with the different methods are compared in Tab. 1, while other high-symmetry adsorption configurations and adsorption data are shown in the Supporting Information.

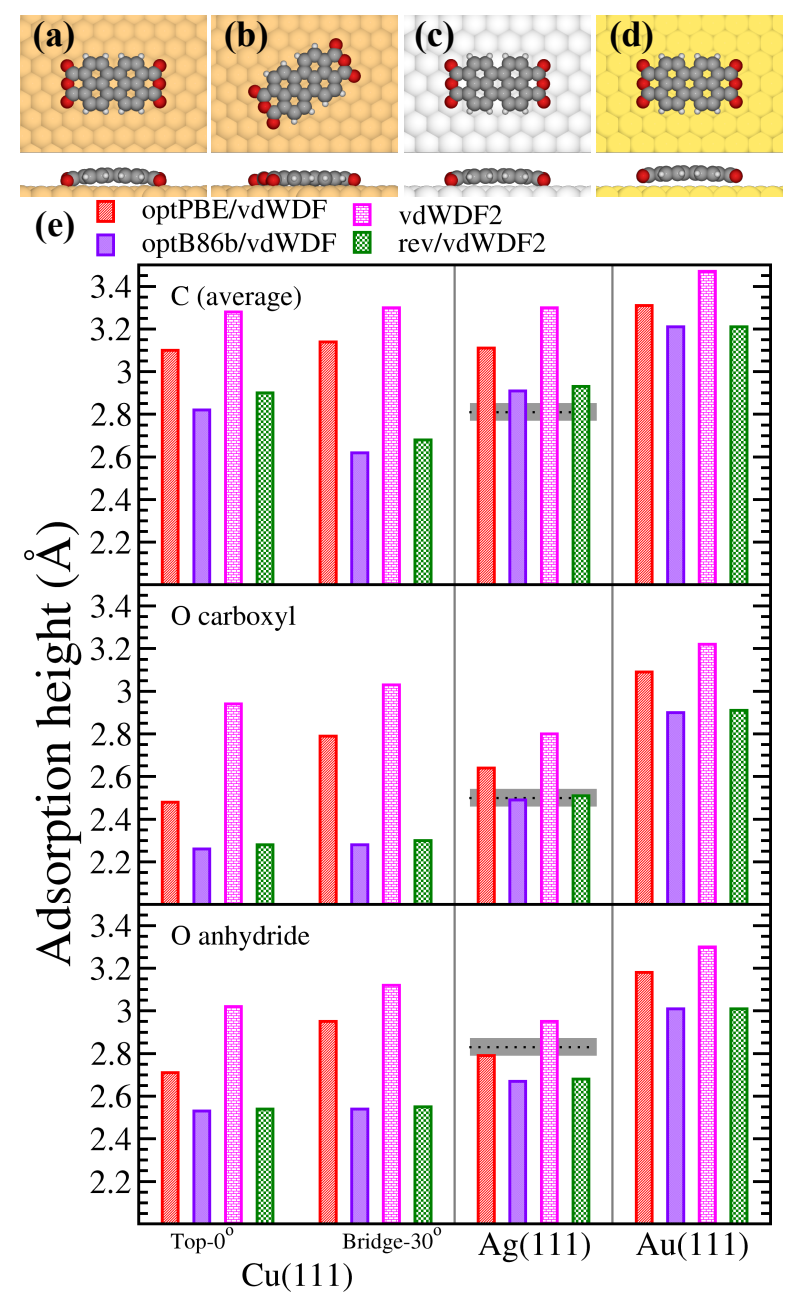

Figure 3 Top and side views for the most favorable adsorption configurations of the isolated PTCDA molecule on (a,b) $\mathrm{Cu}(111),(\mathrm{c}) \mathrm{Ag}(111)$, and (d) $\mathrm{Au}(111)$, as well (e) summary of average adsorption heights of carbons atoms and the two types of oxygen species on the three surfaces obtained with different vdWDFs, as indicated. Our calculated adsorption heights are compared to those obtained experimentally for the $\mathrm{Ag}(111)$ surface. ${ }^{33}$ 
Table 1 Average adsorption heights ( $h_{\text {ads }}$ ) for the carbon and two types of oxygen atoms, as well as adsorption energies $\left(E_{\text {ads }}\right)$ obtained with different vdWDFs, for an isolated PTCDA molecule obtained for different adsorption sites on $\mathrm{Cu}(111), \operatorname{Ag}(111)$, and $\mathrm{Au}(111)$, with two configurations with similar energies indicated for $\mathrm{Cu}(111)$. For $\mathrm{Ag}(111)$, also the adsorption heights from experiments (Exp.) are indicated.

\begin{tabular}{|c|c|c|c|c|}
\hline \multirow{2}{*}{$\begin{array}{l}\text { Substrate and } \\
\text { adsorption site }\end{array}$} & \multicolumn{3}{|c|}{$h_{\text {ads }}(\AA)$} & \multirow[t]{2}{*}{$E_{\text {ads }}(\mathrm{eV})$} \\
\hline & $\mathrm{C}$ average & O carboxyl & O anhydride & \\
\hline \multicolumn{5}{|l|}{$\mathrm{Cu}(111):$ Top- $0^{\circ}$} \\
\hline optPBE/vdWDF & 3.10 & 2.48 & 2.71 & -2.38 \\
\hline optB86b/vdWDF & 2.82 & 2.26 & 2.53 & -3.01 \\
\hline vdWDF2 & 3.28 & 2.94 & 3.02 & -1.72 \\
\hline do rev/vdWDF2 & 2.90 & 2.28 & 2.54 & -1.95 \\
\hline \multicolumn{5}{|c|}{$\mathrm{Cu}(111)$ : Bridge $-30^{\circ}$} \\
\hline optPBE/vdWDF & 3.14 & 2.79 & 2.95 & -2.35 \\
\hline optB86b/vdWDF & 2.62 & 2.28 & 2.54 & -3.04 \\
\hline vdWDF2 & 3.30 & 3.03 & 3.12 & -1.70 \\
\hline $\mathrm{rev} / \mathrm{vdWDF} 2$ & 2.68 & 2.30 & 2.55 & -1.91 \\
\hline \multicolumn{5}{|l|}{$\operatorname{Ag}(111)$} \\
\hline optPBE/vdWDF & 3.11 & 2.64 & 2.79 & -2.55 \\
\hline optB86b/vdWDF & 2.91 & 2.49 & 2.67 & -3.04 \\
\hline vdWDF2 & 3.30 & 2.80 & 2.95 & -1.85 \\
\hline $\mathrm{rev} / \mathrm{vdWDF} 2$ & 2.93 & 2.51 & 2.68 & -2.30 \\
\hline Exp. ${ }^{33}$ & $2.81 \pm 0.04$ & $2.50 \pm 0.04$ & $2.83 \pm 0.04$ & \\
\hline \multicolumn{5}{|l|}{$\operatorname{Au}(111)$} \\
\hline optPBE/vdWDF & 3.31 & 3.09 & 3.18 & -2.42 \\
\hline optB86b/vdWDF & 3.21 & 2.90 & 3.01 & -2.74 \\
\hline vdWDF2 & 3.47 & 3.22 & 3.30 & -1.84 \\
\hline $\mathrm{rev} / \mathrm{vdWDF} 2$ & 3.21 & 2.91 & 3.01 & -2.23 \\
\hline
\end{tabular}


In Fig. 3d the adsorption heights obtained with the different vdWDFs for the most stable configurations, of the isolated PTCDA molecule on the three surfaces, are compared. For $\operatorname{Ag}(111)$ also the measured values are indicated. ${ }^{33}$ For all methods, except optPBE/vdWDF, the adsorption heights follow the established reactivity of the three surfaces, with the smallest adsorption height for $\mathrm{Cu}(111)$ and the largest one for $\mathrm{Au}(111)$. For $\mathrm{Ag}(111)$ the optB86b/vdW and rev/vdWDF2 results compare quite well to the experimental values, with the largest discrepancies for the anhydride oxygen, of $0.16 \AA$ and $0.15 \AA$, respectively. The optPBE/vdWDF and vdWDF2 methods overestimate the heights, except for the anhydride oxygen, which is very well described by optPBE/vdWDF.

Considering the herringbone network of PTCDA, this follows a quite similar construction on all three surfaces, in which one of the two molecules per unit cell adsorbs similar to the most stable adsorption configuration for a single molecule, while the second molecule adopts its geometry in order to optimize the molecule-molecule and molecule-surface interactions, Fig. 4. The structure of the PTCDA herringbone network on these surfaces has been discussed thoroughly elsewhere, ${ }^{23,46}$ and will not be subject for discussion here.

In Fig. 4 the average adsorption heights of the different atomic species for the PTCDA herringbone network for the various vdWDFs are compared to those obtained experimentally, ${ }^{33}$ as well as calculated ones using the DFT+vdW ${ }^{\text {surf }}$ method by Ruiz et al. ${ }^{23}$ Additional adsorption data is listed in Tab. 2 As a general remark, the adsorption heights are larger in the network compared to the isolated molecules, due to the intermolecular interactions. Notably, similar effects have been observed for other systems. ${ }^{48}$ Considering the average height of the carbon atoms, these compare extraordinarily well to the experimental data for optB86b/vdWDF and rev/vdWDF, with perfect agreement for $\mathrm{Cu}(111)$, while both methods give slightly overestimated and underestimated heights on $\mathrm{Ag}(111)$ and $\mathrm{Au}(111)$, respectively, with less than $0.1 \AA$. The optPBE and vdWDF2 methods severely overestimates the heights of the carbon atoms, with exception of optPBE/vdWDF for $\mathrm{Au}(111)$ where a reasonable agreement is found. 

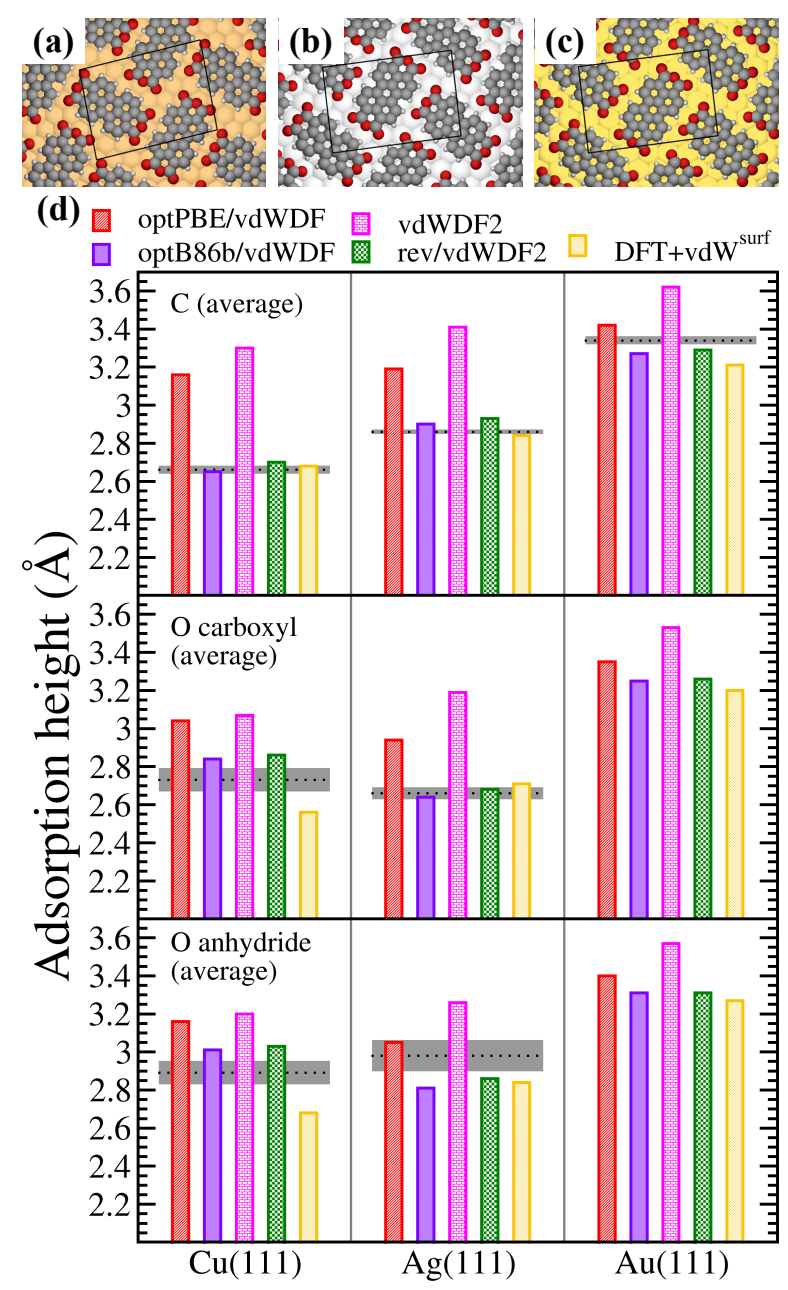

Figure 4 Most favorable configurations of the PTCDA herringbone structure on (a) $\mathrm{Cu}(111)$, (b) $\mathrm{Ag}(111)$, and (c) $\mathrm{Au}(111)$, with the black lines indicating the surface unit cells, and (d) summary of average adsorption heights of these structures. The average adsorption heights were calculated for the carbon atoms and the two types of oxygen atoms with different vdWDFs, and compared to literature values obtained with the DFT $+v d W^{\text {surf }}$ method. ${ }^{23}$ The dotted black lines and shaded areas denote the experimental data and associated error bars, for $\mathrm{Cu}(111),{ }^{34} \mathrm{Ag}(111),{ }^{33}$, and $\mathrm{Au}(111)^{35}$ (only carbon atoms). 
Table 2 Average adsorption heights ( $h_{\text {ads }}$ ) for the carbon and two types of oxygen atoms, as well as adsorption energies $\left(E_{\text {ads }}\right)$ obtained with different vdWDFs, for the herringbone structure of PTCDA obtained for the most stable adsorption configurations on $\mathrm{Cu}(111), \mathrm{Ag}(111)$, and $\mathrm{Au}(111)$. Where available, also the adsorption heights from experiments (Exp.) are indicated.

\begin{tabular}{|c|c|c|c|c|}
\hline & \multicolumn{3}{|c|}{$h_{\text {ads }}(\AA)$} & \multirow[t]{2}{*}{$E_{\text {ads }}(\mathrm{eV})$} \\
\hline & $\mathrm{C}$ average & O carboxyl & O anhydride & \\
\hline \multicolumn{5}{|l|}{$\mathrm{Cu}(111)$} \\
\hline optPBE/vdWDF & 3.16 & 3.04 & 3.16 & -2.95 \\
\hline optB86/vdWDF & 2.65 & 2.84 & 3.01 & -3.49 \\
\hline vdWDF2 & 3.30 & 3.07 & 3.20 & -2.29 \\
\hline $\mathrm{rev} / \mathrm{vdWDF} 2$ & 2.70 & 2.86 & 3.03 & -2.81 \\
\hline Exp. ${ }^{34}$ & $2.66 \pm 0.02$ & $2.73 \pm 0.06$ & $2.89 \pm 0.06$ & \\
\hline \multicolumn{5}{|l|}{$\operatorname{Ag}(111)$} \\
\hline optPBE/vdWDF & 3.19 & 2.94 & 3.05 & -3.22 \\
\hline optB86b/vdWDF & 2.90 & 2.64 & 2.81 & -3.73 \\
\hline vdWDF2 & 3.41 & 3.19 & 3.26 & -2.37 \\
\hline rev/vdWDF2 & 2.93 & 2.68 & 2.86 & -3.06 \\
\hline Exp. ${ }^{33}$ & $2.86 \pm 0.01$ & $2.66 \pm 0.03$ & $2.98 \pm 0.08$ & \\
\hline \multicolumn{5}{|l|}{$\operatorname{Au}(111)$} \\
\hline optPBE/vdWDF & 3.42 & 3.35 & 3.40 & -3.26 \\
\hline optB86b/vdWDF & 3.27 & 3.25 & 3.31 & -3.52 \\
\hline vdWDF2 & 3.62 & 3.53 & 3.57 & -2.52 \\
\hline $\mathrm{rev} / \mathrm{vdWDF} 2$ & 3.29 & 3.26 & 3.31 & -2.97 \\
\hline Exp. ${ }^{35}$ & $3.34 \pm 0.02$ & - & - & \\
\hline
\end{tabular}


In view of the oxygen atoms, the comparison to experiments is limited to $\mathrm{Cu}(111)$ and $\mathrm{Ag}(111)$, as no experimental data exist for $\mathrm{Au}(111)$. The optB86b/vdWDF and rev/vdWDF2 calculations are able to capture most of the trends for these atoms, with exception of the anhydride oxygen on $\operatorname{Ag}(111)$, which we find at a height closer to the surface than the carbon atoms, while the opposite is observed experimentally. ${ }^{33}$ The carboxyl oxygens, on the other hand, are found in almost perfect agreement with the measurements using these methods for this surface. The same behavior was found for the isolated PTCDA molecule on this surface (Fig. 3) On $\mathrm{Cu}(111)$ both the types of oxygen atoms are found at approximately $0.1 \AA$ too far away from the surface for optB86b/vdWDF and rev/vdWDF2 compared to experiments. As for the carbon atoms, optPBE/vdWDF and vdWDF2 overestimate the adsorption heights quite significantly.

Comparing the data to DFT+vdW ${ }^{\text {surf }}$, both optB86b/vdWDF and rev/vdWDF2 are in better, or equally good, agreement to experiments with only one exception; the carbon atoms on $\operatorname{Ag}(111)$. It should, however, be noted that in most cases, the three methods are in close agreement with experiments, and up to this point, all three methods appear as suitable choices. It is important to notice that these methods are able to describe the adsorption heights of the carbon atoms with higher accuracy than those of the oxygen atoms.

The herringbone network on $\mathrm{Cu}(111)$ warrants additional analysis. In the experiments, the carbon atoms are found closer to the surface than the oxygen atoms, in contrast to $\operatorname{Ag}(111)$ and $\mathrm{Cu}(111)$. Notable, both optB86b/vdWDF and rev/vdWDF2 are able to qualitatively reproduce this phenomenon, although the oxygen atoms are found slightly too far away from the surface for both functionals. Notable, for the isolated PTCDA on $\mathrm{Cu}(111)$ the anticipated adsorption behavior, with the oxgen atoms closer to the surface than the carbon atoms, is found. We ascribe this behavior to two competing attractive interactions between PTCDA and the $\mathrm{Cu}(111)$ surface, namely the interaction of the surface with the oxygen atoms and the perylene core, respectively. For the isolated molecule, the oxygencopper interaction is the stronger of the two. In the formation of the network this interaction is weaken as a result of the intermolecular interactions, just enough to make the interaction between carbon and copper the dominating molecule-surface interaction. Similar to the adsorption of DIP on this surface (see below) PTCDA is adsorbed in a bent configuration pushing the oxygen atoms away from the surface. 


\subsection{Adsorption of DIP}

The adsorption of DIP on the (111)-facets of the noble metals has previously been investigated with DFT by Bürker et al. ${ }^{36}$ using the DFT+vdW ${ }^{\text {surf }}$ method. However, in their study a specific adsorption site was assumed, without considering other possible sites. Thus, the exact adsorption configurations of DIP on the three surfaces are unknown.

Fig. 5 illustrates the most stable adsorption sites of an isolated DIP molecule adsorbed on the three surfaces, respectively, as obtained with optB86b/vdWDF. All high-symmetry adsorption sites are compared in the Supporting Information. The preferred adsorption site of DIP is the same on all surfaces, with the molecule centered above a bridge site with a $30^{\circ}$ angle between the long axis of the molecule and the [1ㅣㅣ direction of respective substrate. On the $\operatorname{Ag}(111)$ and $\mathrm{Au}(111)$ surfaces the molecule adsorb in a more or less flat geometry, while on $\mathrm{Cu}(111)$ the molecule is considerably bent, with the centre of the molecule closest to the surface, similar to the adsorption site of PTCDA in the herringbone network on $\mathrm{Cu}(111)$ (see above). Notably, the adsorption sites we found are different from those used by Bürker et al., ${ }^{36}$ where it was assumed that the molecule adsorbs centered above a top site, parallel to the [110] for each of the surfaces. The impact of the surface registry is discussed below.

Considering the adsorption heights of the most stable adsorption configurations, these compare very well to experimental data in the literature for optB86b/vdWDF and rev/vdWDF2, as shown in Fig. 5d and Tab. 3 (including additional adsorption data). Strikingly, both methods reproduce the experiments close to a precision of $0.1 \AA$, where the largest disagreement is found for the optB86b/vdWDF$\operatorname{Ag}(111)$ combination, with an underestimation of $0.11 \AA$ A . As for PTCDA, optPBE/vdWDF and vdWDF2 give a severe overestimation of the adsorption heights, except for $\mathrm{Au}(111)$ where a reasonable agreement is found. In Fig. $5 \mathrm{~d}$ also the adsorption heights obtained with the DFT+vdW ${ }^{\text {surf }}$ method, ${ }^{23}$ as reported by Bürker et al. ${ }^{36}$, are shown. However, the direct comparison is less than trivial as these were not obtained for the most stable adsorption configuration, as noted above. Thus, before making an assessment of the two methods we need to analyze how much the adsorption height differs between different adsorption configurations. 

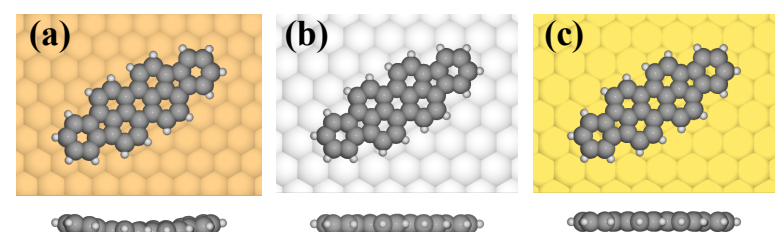

conorespo
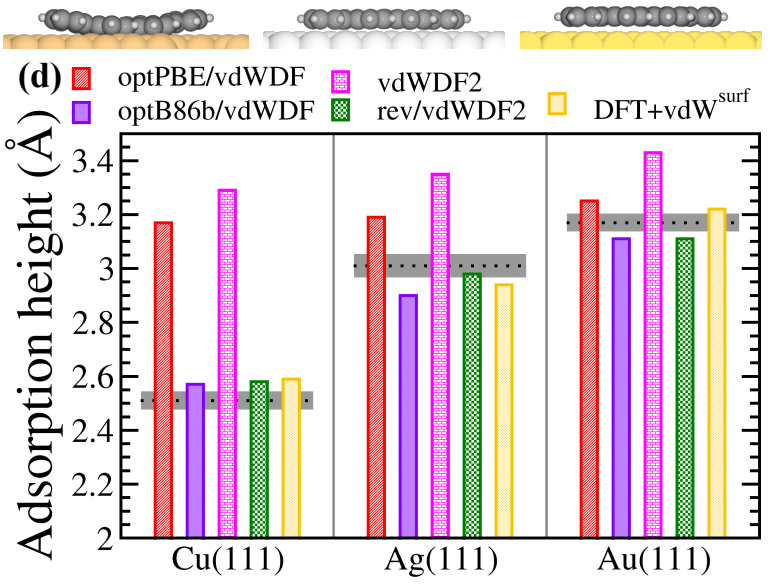

Figure 5 Top and side views of the most favorable adsorption configurations of DIP on (a) $\mathrm{Cu}(111)$, (b) $\operatorname{Ag}(111)$, and (c) $\mathrm{Au}(111)$, and (d) summary of average adsorption heights on the three surfaces, calculated with different vdWDFs. The dotted black lines and shaded areas denote the experimental data and associated error bars. ${ }^{36}$ The DFT+vdW ${ }^{\text {surf }}$ data were extracted from Ref. ${ }^{36}$, however, these data do not correspond to the most stable adsorption configurations of DIP on the three surfaces, the consequence of which is discussed in the main text. 
Table 3 Average, maximum and minimum adsorption heights $\left(h_{\mathrm{ads}}\right)$ for the carbon atoms, as well as adsorption energies $\left(E_{\text {ads }}\right)$ obtained with different vdWDFs, for DIP obtained for different adsorption sites on $\mathrm{Cu}(111), \mathrm{Ag}(111)$, and $\mathrm{Au}(111)$, with the results of the most stable adsorption sites italicized. For each of the surface, the average experimental (Exp.) adsorption height from Ref. ${ }^{36}$ is indicated.

\begin{tabular}{lllll}
\hline $\begin{array}{l}\text { Substrate and } \\
\text { adsorption site }\end{array}$ & \multicolumn{3}{c}{$h_{\text {ads }}(\AA)$} & \multicolumn{2}{c}{$E_{\text {ads }}(\mathrm{eV})$} \\
\hline $\begin{array}{l}\text { Cu(111) } \\
\text { optPBE/vdWDF }\end{array}$ & 3.17 & Max. & Min. & \\
optB86b/vdWDF & 2.57 & 3.23 & 3.12 & -2.81 \\
vdWDF2 & 3.29 & 3.31 & 3.21 & -2.01 \\
rev/vdWDF2 & 2.58 & 3.06 & 2.33 & -2.89 \\
Exp. & & & & \\
& $2.51 \pm 0.03$ & & &
\end{tabular}

$\operatorname{Ag}(111)$

$\begin{array}{lllll}\text { optPBE/vdWDF } & 3.19 & 3.21 & 3.14 & -2.73 \\ \text { optB86b/vdWDF } & 2.90 & 2.94 & 2.88 & -3.30 \\ \text { vdWDF2 } & 3.35 & 3.41 & 3.28 & -1.99 \\ \text { rev/vdWDF2 } & 2.98 & 3.00 & 2.96 & -2.52 \\ \text { Exp. }^{36} & 3.01 \pm 0.04 & & & \end{array}$

$\operatorname{Au}(111)$

\begin{tabular}{lllll} 
optPBE/vdWDF & 3.25 & 3.31 & 3.17 & -3.06 \\
optB86b/vdWDF & 3.11 & 3.13 & 3.06 & -3.57 \\
vdWDF2 & 3.43 & 3.49 & 3.35 & -2.33 \\
rev/vdWDF2 & 3.11 & 3.14 & 3.07 & -2.99 \\
Exp. $^{36}$ & $3.17 \pm 0.03^{a}$ & & & \\
\hline
\end{tabular}

${ }^{a}$ This value is reduced to $3.10 \AA$ if taking the surface reconstruction of $\mathrm{Au}(111)$ into account. ${ }^{36}$ 
Table 4 summarizes adsorption height averages, maxima and minima for the Top $-0^{\circ}$ and Bridge$30^{\circ}$ adsorption sites, as well as the binding energies, calculated with optB86b/vdWDF. The geometries are named by the position of the DIP center over the surface and the rotation of the molecule's long axis away from the [1ํㅣㅇ-direction of respective substrate. Depictions of each of the geometries can be found in the Supporting Information. As mentioned above, the Bridge- $30^{\circ}$ is the most stable adsorption configuration on all surfaces, while the DFT+vdW ${ }^{\text {surf }}$ results in Fig. 5 were obtained for the Top- $0^{\circ}$ geometry. The difference of the average adsorption height between these configurations is about $0.1 \AA$ for $\mathrm{Cu}(111)$ and $\mathrm{Ag}(111)$. Considering the similar results for optB86b/vdWDF and $\mathrm{DFT}+\mathrm{vdW}$ surf , we estimate that the adsorption heights for the latter is overestimated by roughly $0.1 \AA$, as a result of using the wrong adsorption geometry. For $\mathrm{Au}(111)$ the difference between the two sites is as small as $0.03 \AA$, thus the adsorption height from DFT+vdW $\mathrm{W}^{\text {surf }}$ is only slightly overestimated. Following this tentative analysis, it appears that the both method performs equally well in describing the adsorption height of the DIP molecule.

Table 4 Average, maximum and minimum adsorption heights $\left(h_{\mathrm{ads}}\right)$ for the carbon atoms, as well as adsorption energies $\left(E_{\mathrm{ads}}\right)$, obtained with optB86b/vdWDF for DIP obtained for different adsorption sites on $\mathrm{Cu}(111), \mathrm{Ag}(111)$, and $\mathrm{Au}(111)$, with the results of the most stable adsorption sites italicized. For each of the surface, the average experimental (Exp.) adsorption height from Ref. ${ }^{36}$ is indicated.

\begin{tabular}{|c|c|c|c|c|}
\hline \multirow{2}{*}{$\begin{array}{l}\text { Substrate and } \\
\text { adsorption site }\end{array}$} & \multicolumn{3}{|c|}{$h_{\text {ads }}(\AA)$} & \multirow[t]{2}{*}{$E_{\text {ads }}(\mathrm{eV})$} \\
\hline & Average & Max. & Min. & \\
\hline \multicolumn{5}{|l|}{$\mathrm{Cu}(111)$} \\
\hline do Top- $-0^{\circ}$ & 2.69 & 2.88 & 2.53 & -3.84 \\
\hline do Bridge $-30^{\circ}$ & 2.58 & 3.06 & 2.31 & -3.89 \\
\hline
\end{tabular}

$\operatorname{Ag}(111)$

$\begin{array}{lllll}\text { do Top- } 0^{\circ} & 3.00 & 3.17 & 2.91 & -3.24 \\ \text { do Bridge-30 } & 2.89 & 2.93 & 2.85 & -3.43\end{array}$

\section{$\operatorname{Au}(111)$}

\begin{tabular}{lllll} 
do Top- $0^{\circ}$ & 3.14 & 3.22 & 3.06 & -3.44 \\
do Bridge-30 & 3.11 & 3.13 & 3.07 & -3.57 \\
\hline
\end{tabular}




\section{Discussion}

In Tab. 5 the mean absolute errors (MAEs) of the calculated adsorption heights from the different methods method, with respect to experimental literature values, ${ }^{33-36}$ are summarized. From the results, it is clear that the choice of functional is more important for the more interacting surfaces. This is reflected by the fact that adsorption on $\mathrm{Au}(111)$ is reasonably described by all methods, with the exception of vdWDF2, while the accuracy on $\mathrm{Cu}(111)$ diverges quite significantly between the functionals. This may be coupled to that synergy effects between van der Waals and chemical interactions ${ }^{49}$ are more prominent for the chemically active $\mathrm{Cu}(111)$ surface compared to the inert $\mathrm{Au}(111)$ surface, rendering the results more sensitive to the $\mathrm{XC}$-functional for the former. In any case, the optB86b/vdWDF and rev/vdWDF2 outperform the other tested functionals independent on the system under study. For both these functionals, it is found that the carbon atoms are described slightly better than oxygen atoms, although both functionals give MAEs within $0.1 \AA$ for both types of atoms. Considering the different surfaces, the MAEs are more or less the same, with the exception for the carbon atoms on $\mathrm{Cu}$ described by optB86b/vdWDF, where it is as small as $(0.04 \pm 0.02) \AA$. This is in line with recent results for the optB86b/vdWDF functional, ${ }^{31}$ where an MAE of $(0.05 \pm 0.05) \AA$ was found for a perylene derivative both in its isolated form and in a 2D metal organic framework, adsorbed on this surface. The apparent generality of the optB86b/vdWDF method, in describing adsorption heights for large organic molecules on metals, makes it a promising choice for theoretical modeling of this type of systems, within the DFT level of theory, as is the rev/vdWDF2 functional. Notably, with the available data for PTCDA and DIP at hand, also the DFT $+v d W^{\text {surf }}$ method presents a propitious method. Moreover, this method describes the adsorption of benzene on $\operatorname{Pt}(111)$ and $\mathrm{Au}(111)$ with an

accuracy of $0.1 \AA .{ }^{49}$ Before we can conclude which of the three methods is the most rigorous one, the comparison needs to be expanded to include also other molecules and surfaces. 
Table 5 Mean absolute errors of the calculated adsorption heights from the different vdWDFs, compared to experimentally measured heights, for PTCDA and DIP adsorbed on $\mathrm{Cu}(111), \operatorname{Ag}(111)$, and $\mathrm{Au}(111)$. The data is shown for both carbon and oxygen atoms and units are given in $\AA$.

\begin{tabular}{llll}
\hline & Carbon atoms & Oxygen atom & All atoms \\
\hline $\mathbf{C u}(\mathbf{1 1 1})$ & & & \\
optPBE/vdWDF & $0.58 \pm 0.02$ & $0.29 \pm 0.06$ & $0.44 \pm 0.04$ \\
optB86b/vdWDF & $0.04 \pm 0.02$ & $0.12 \pm 0.06$ & $0.08 \pm 0.04$ \\
vdWDF2 & $0.78 \pm 0.02$ & $0.33 \pm 0.06$ & $0.55 \pm 0.04$ \\
rev/vdWDF2 & $0.06 \pm 0.02$ & $0.14 \pm 0.06$ & $0.10 \pm 0.04$
\end{tabular}

$\operatorname{Ag}(111)$

$\begin{array}{llll}\text { optPBE/vdWDF } & 0.27 \pm 0.03 & 0.11 \pm 0.05 & 0.19 \pm 0.04 \\ \text { optB86b/vdWDF } & 0.08 \pm 0.03 & 0.09 \pm 0.05 & 0.09 \pm 0.04 \\ \text { vdWDF2 } & 0.46 \pm 0.03 & 0.31 \pm 0.05 & 0.37 \pm 0.04 \\ \text { rev/vdWDF2 } & 0.07 \pm 0.03 & 0.08 \pm 0.05 & 0.07 \pm 0.04\end{array}$

$\operatorname{Au}(111)$

$\begin{array}{llll}\text { optPBE} / \mathrm{vdWDF} & 0.08 \pm 0.03 \quad- & 0.08 \pm 0.03\end{array}$

$\begin{array}{llll}\mathrm{optB} 86 \mathrm{~b} / \mathrm{vdWDF} & 0.07 \pm 0.03 \quad- & 0.07 \pm 0.03\end{array}$

$\begin{array}{llll}\mathrm{vdWDF} 2 & 0.27 \pm 0.03 \quad- & 0.27 \pm 0.03\end{array}$

$\begin{array}{llll}\mathrm{rev} / \mathrm{vdWDF} 2 & 0.06 \pm 0.03 \quad- & 0.06 \pm 0.03\end{array}$

\begin{tabular}{llll} 
All surfaces & & & \\
optPBE/vdWDF & $0.30 \pm 0.03$ & $0.19 \pm 0.05$ & $0.25 \pm 0.04$ \\
optB86b/vdWDF & $0.06 \pm 0.03$ & $0.10 \pm 0.05$ & $0.08 \pm 0.04$ \\
vdWDF2 & $0.50 \pm 0.03$ & $0.31 \pm 0.05$ & $0.41 \pm 0.04$ \\
rev/vdWDF2 & $0.06 \pm 0.03$ & $0.10 \pm 0.05$ & $0.08 \pm 0.04$ \\
\hline
\end{tabular}


Having illustrated that the optB86b/vdWDF and rev/vdWDF2 functionals are able to describe adsorption heights with high accuracy, the next step in understanding how the functionals affect the adsorption characteristics is to study the adsorption energies. It has been illustrated ${ }^{50}$ that the optB86b/vdWDF functional overestimates the adsorption strength for graphene on $\mathrm{Ni}$, while accurately describing the adsorption height. There are indications that the adsorption energy is overestimated also for other systems. ${ }^{51}$ On the other hand, rev/vdWDF2 has been shown to accurately describe the adsorption energy of benzene on $\mathrm{Cu}(111) .{ }^{41}$ Notably rev/vdWDF2 gives considerably smaller adsorption energies than optB86b/vdWDF for the systems studies here, however, a direct comparison of adsorption energies is not possible due to the absence of reliable benchmark data. The results of rev/vdWDF2 are nevertheless interesting, as it shows the capability of describing structural properties equally accurate as optB86b/vdWDF, at the same time as lowering the depth of the interaction potential. This illustrates the importance to both optimize the exchange and correlation term when designing new non-local density functionals. We expect that the improved description of the adsorption of large organic molecules on metals will at first hand come from the development of new density functionals. For this to become possible additional experimental benchmarks are needed, in particular accurate and reliable measurements of adsorption energies. In the long term, we may even envision that calculations with the random phase approximation ${ }^{52}$ will become possible do describe the type of systems studied here, however, only when the available computational resources have increased significantly.

We would like to stress that accurate description of adsorption energies is of secondary importance compared to the adsorption heights. Precise determination of material characteristics, such as change transfer in a metal-organic contact, ${ }^{53}$ rather rely on the structural properties, and is not dependent on the adsorption energy. Also for the description of reactions on surfaces, the adsorption height is the most crucial property for determining how a metal catalyze a reaction. For on-surface reactions, the energy barriers rely on energy difference between adsorbed species, where systematic errors in the adsorption energies to a large extent are canceled out, if the molecules are positioned at similar adsorption heights in the different reaction states. Therefore, under these conditions, we expect that both the optB86b/vdWDF and the rev/vdWDF2 functionals will accurately describe reaction between organic molecules on surface. 


\section{Conclusion}

To conclude, we have investigated how well various van der Waals density functionals describe the adsorption heights of organic molecules on coinage metal surface, presenting very encouraging results for the optB86b/vdWDF and rev/vdWDF2 functionals. With only few exceptions, these functionals describe adsorption heights within $0.1 \AA$, both with an average error of $(0.06 \pm 0.03) \AA$ for carbon atoms. Within the DFT level of theory, these functionals are highly recommended choices for describing properties and processes for large organic molecules adsorbed on metal surfaces. The ability of DFT to provide reliable description for large organic molecules on metals is envisioned to play a vital role for the movement towards in silico designed molecular frameworks on metals, with tailor-made characteristics.

\section{Acknowledgements}

Dr. Meike Stöhr at Zernike Institute, Groningen, is acknowledged for sharing her expertise about X-ray standing wave measurements. Funding was provided by the Swedish Research Council. Com-

puter resources were allocated at the National Supercomputer Centre, Swede, through SNAC and the MATTER consortium.

\section{References}

[1] Stöhr, M.; Wahl, M.; Galka, C. H.; Riehm, T.; Jung, T. a.; Gade, L. H. Angew. Chem. Int. Ed. 2005, 44, 7394-7398.

[2] Schlickum, U.; Decker, R.; Klappenberger, F.; Zoppellaro, G.; Klyatskaya, S.; Ruben, M.; Silanes, I.; Arnau, A.; Kern, K.; Brune, H.; Barth, J. V. Nano Lett. 2007, 7, 3813-3817.

[3] Staniec, P. A.; Perdigão, L. M. A.; Rogers, B. L.; Champness, N. R.; Beton, P. H. J. Phys. Chem. C 2007, 111, 886-893.

[4] Klappenberger, F.; Kühne, D.; Krenner, W.; Silanes, I.; Arnau, A.; García de Abajo, F.; Klyatskaya, S.; Ruben, M.; Barth, J. V. Phys. Rev. Lett. 2011, 106, 026802-026805. 
[5] Stöhr, M.; Boz, S.; Schär, M.; Nguyen, M.-T.; Pignedoli, C. A.; Passerone, D.; Schweizer, W. B.; Thilgen, C.; Jung, T. A.; Diederich, F. Angew. Chem. Int. Ed. 2011, 50, 9982-9986.

[6] Grill, L.; Dyer, M.; Lafferentz, L.; Persson, M.; Peters, M. V.; Hecht, S. Nature Nanotech. 2007, 2, 687-691.

[7] Cai, J.; Ruffieux, P.; Jaafar, R.; Bieri, M.; Braun, T.; Blankenburg, S.; Muoth, M.; Seitsonen, A. P.; Saleh, M.; Feng, X.; Müllen, K.; Fasel, R. Nature 2010, 466, 470-473.

[8] Franc, G.; Gourdon, A. Phys. Chem. Chem. Phys. 2011, 13, 14283-14292.

[9] Bieri, M.; Blankenburg, S.; Kivala, M.; Pignedoli, C. A.; Ruffieux, P.; Müllen, K.; Fasel, R. Chem. Commun. 2011, 47, 10239-10241.

[10] Zhang, Y.-Q.; Kepčija, N.; Kleinschrodt, M.; Diller, K.; Fischer, S.; Papageorgiou, A. C.; Allegretti, F.; Björk, J.; Klyatskaya, S.; Klappenberger, F.; Ruben, M.; Barth, J. V. Nat. Commun. 2012, 3, 1286.

[11] Haq, S.; Hanke, F.; Dyer, M. S.; Persson, M.; Iavicoli, P.; Amabilino, D. B.; Raval, R. J. Am. Chem. Soc. 2011, 133, 12031-12039.

[12] Bieri, M.; Nguyen, M.-T.; Gröning, O.; Cai, J.; Treier, M.; Aït-Mansour, K.; Ruffieux, P.; Pignedoli, C. A.; Passerone, D.; Kastler, M.; Müllen, K.; Fasel, R. J. Am. Chem. Soc. 2010, $132,16669-16676$.

[13] Treier, M.; Pignedoli, C. A.; Laino, T.; Rieger, R.; Müllen, K.; Passerone, D.; Fasel, R. Nature Chem. 2011, 3, 61-67.

[14] Nguyen, M.-T.; Pignedoli, C. A.; Passerone, D. Phys. Chem. Chem. Phys. 2011, 13, 154-160.

[15] Björk, J.; Hanke, F.; Stafström, S. J. Am. Chem. Soc. 2013, 135, 5768-5775.

[16] Björk, J.; Hanke, F. Chem. Eur. J. 2014, 20, 928-934.

[17] Jacobsen, C. J.; Dahl, S.; Clausen, B. S.; Bahn, S.; Logadottir, A.; Nø rskov, J. K. J. Am. Chem. Soc. 2001, 123, 8404-8405. 
[18] Mura, M.; Gulans, A.; Thonhauser, T.; Kantorovich, L. Phys. Chem. Chem. Phys. 2010, 12, 4759-4767.

[19] Grimme, S. J. Comp. Chem. 2006, 27, 1787-1799.

[20] Tkatchenko, A.; Scheffler, M. Phys. Rev. Lett. 2009, 102, 073005-073008.

[21] Hanke, F. J. Comp. Chem. 2011, 32, 1424-1430.

[22] McNellis, E. R.; Meyer, J.; Reuter, K. Phys. Rev. B 2009, 80, 205414-205423.

[23] Ruiz, V.; Liu, W.; Zojer, E.; Scheffler, M.; Tkatchenko, A. Phys. Rev. Lett. 2012, 108, 146103146107.

[24] Dion, M.; Rydberg, H.; Schröder, E.; Langreth, D. C.; Lundqvist, B. I. Phys. Rev. Lett. 2004, 92, 246401-246404.

[25] Zhang, Y.; Yang, W. Phys. Rev. Lett. 1998, 80, 890.

[26] Hanke, F.; Dyer, M. S.; Björk, J.; Persson, M. J. Phys. Condens. Matt. 2012, 24, 424217-424225.

[27] Lee, K.; Murray, E. D.; Kong, L.; Lundqvist, B. I.; Langreth, D. C. Phys. Rev. B 2010, 82, 081101(R)-081104(R).

[28] Klimeš, J.; Bowler, D. R.; Michaelides, A. J. Phys. Condens. Matt. 2010, 22, 022201-022205.

[29] Klimeš, J.; Bowler, D. R.; Michaelides, A. Phys. Rev. B 2011, 83, 195131-195143.

[30] Cooper, V. R. Phys. Rev. B 2010, 81, 161104(R)-161107(R).

[31] Matena, M.; Björk, J.; Wahl, M.; Lee, T.-L.; Zegenhagen, J.; Gade, L. H.; Jung, T. A.; Persson, M.; Stöhr, M. Submitted for publication 2014,

[32] Lüder, J.; Sanyal, B.; Eriksson, O.; Puglia, C.; Brena, B. Phys. Rev. B 2014, 89, 045416-045427.

[33] Hauschild, A.; Temirov, R.; Soubatch, S.; Bauer, O.; Schöll, A.; Cowie, B. C. C.; Lee, T.-L.; Tautz, F. S.; Sokolowski, M. Phys. Rev. B 2010, 81, 125432-125443. 
[34] Gerlach, A.; Sellner, S.; Schreiber, F.; Koch, N.; Zegenhagen, J. Phys. Rev. B 2007, 75, 045401045407.

[35] Henze, S.; Bauer, O.; Lee, T.-L.; Sokolowski, M.; Tautz, F. Surf. Sci. 2007, 601, 1566-1573.

[36] Bürker, C.; Ferri, N.; Tkatchenko, A.; Gerlach, A.; Niederhausen, J.; Hosokai, T.; Duhm, S.; Zegenhagen, J.; Koch, N.; Schreiber, F. Phys. Rev. B 2013, 87, 165443-165447.

[37] Kresse, G.; Furthmüller, J. Phys. Rev. B 1996, 54, 11169-11186.

[38] Bahn, S. R.; Jacobsen, K. W. Comp. Sci. Eng. 2002, 4, 56-66.

[39] Blöchl, P. E. Phys. Rev. B 1994, 50, 17953-17979.

[40] Kresse, G.; Joubert, D. Phys. Rev. B 1999, 59, 1758-1775.

[41] Hamada, I. Phys. Rev. B 2014, 89, 121103.

[42] Kilian, L.; Umbach, E.; Sokolowski, M. Surf. Sci. 2004, 573, 359-378.

[43] Fenter, P.; Schreiber, F.; Zhou, L.; Eisenberger, P.; Forrest, S. Phys. Rev. B 1997, 56, 3046-3053.

[44] Schmitz-Hübsch, T.; Fritz, T.; Sellam, F.; Staub, R.; Leo, K. Phys. Rev. B 1997, 55, 7972-7976.

[45] Kilian, L.; Umbach, E.; Sokolowski, M. Surf. Sci. 2006, 600, 2633-2643.

[46] Romaner, L.; Nabok, D.; Puschnig, P.; Zojer, E.; Ambrosch-Draxl, C. New J. Phys 2009, 11, 053010.

[47] Wagner, T.; Bannani, A.; Bobisch, C.; Karacuban, H.; Möller, R. J. Phys. Condens. Matt. 2007, $19,056009$.

[48] Simpson, S.; Kunkel, D. a.; Hooper, J.; Nitz, J.; Dowben, P. a.; Routaboul, L.; Braunstein, P.; Doudin, B.; Enders, A.; Zurek, E. J. Phys. Chem. C 2013, 117, 16406-16415.

[49] Liu, W.; Carrasco, J.; Santra, B.; Michaelides, A.; Scheffler, M.; Tkatchenko, A. Phys. Rev. B 2012, 86, 245405-245410. 
[50] Mittendorfer, F.; Garhofer, A.; Redinger, J.; Klimeš, J.; Harl, J.; Kresse, G. Phys. Rev. B 2011, 84, 201401-201404.

[51] Kepčija, N.; Zhang, Y.-Q.; Kleinschrodt, M.; Björk, J.; Klyatskaya, S.; Klappenberger, F.; Ruben, M.; Barth, J. V. J. Phys. Chem. C 2013, 117, 3987-3995.

[52] Schimka, L.; Harl, J.; Stroppa, A.; Grüneis, A.; Marsman, M.; Mittendorfer, F.; Kresse, G. Nature Mater. 2010, 9, 741-744.

[53] Medeiros, P. V. C.; Gueorguiev, G. K.; Stafström, S. Phys. Rev. B 2012, 85, 205423-205429. 